\title{
CARACTERÍSTICAS ACÚSTICAS DA OCLUSIVA GLOTAL ASSOCIADA À SEQUÊNCIA DE PIERRE ROBIN: ESTUDO DE CASO
}

\author{
Acoustic characteristics of glottal stop associated to \\ Pierre Robin Sequence: Case study
}

\author{
Viviane Cristina de Castro Marino ${ }^{(1)}$, Larissa Cristina Berti (2), Aveliny Mantovan Lima-Gregio (3)
}

\begin{abstract}
RESUMO
A fissura de palato, em associação à Sequência de Pierre Robin, pode favorecer o desenvolvimento de produções atípicas (compensatórias), na fala da criança, como é o caso da oclusiva glotal (golpe de glote) comumente observada em substituição aos sons oclusivos (vozeados ou não). No presente estudo, foi realizada a análise dos parâmetros fonético-acústicos da oclusiva glotal produzidas em /k/ e /g/ por uma criança do gênero feminino, com 5 anos, que apresentava fissura de palato reparada, associada à Sequência de Pierre Robin. Para isso, foram selecionadas seis palavras em que a oclusiva velar encontrava-se na posição inicial da palavra e combinada com as vogais /a/, /i/ e /u/ na posição acentuada. Foi ainda realizado julgamento perceptivo-auditivo por três fonoaudiólogos, que apresentou concordância quanto à presença da oclusiva glotal de 100\% para ambas as relações (intra e inter-juízes). Na inspeção dos dados via espectrograma foi observada variabilidade dos parâmetros espectrais (burst e transição formântica) e essas variações também puderam ser computadas considerando as vogais separadamente. A análise estatística revelou diferença estatisticamente significante entre as duas consoantes velares (/k/ e $/ \mathrm{g} /$ ) nos parâmetros espectral (burst), temporal (VOT e duração relativa da oclusiva na palavra) e os relativos às características acústicas das vogais adjacentes às oclusivas (período estacionário de F3). Por fim, as características acústicas da oclusiva glotal sugeriram que a criança pode ter utilizado de estratégias para marcar contrastes fônicos na língua, ainda que os mesmos não tenham magnitude suficiente para serem resgatados auditivamente pelo ouvinte.
\end{abstract}

DESCRITORES: Acústica da Fala; Espectrografia; Distúrbios da Fala; Fissura Palatina; Síndrome de Pierre Robin

(1) Fonoaudióloga; Docente vinculada ao Departamento de Fonoaudiologia da Universidade Estadual Paulista UNESP, Campus de Marília; Doutora em Communication Sciences and Disorders pela Universidade da Florida, EUA.

(2) Fonoaudióloga; Docente e Pesquisadora vinculada ao Departamento de Fonoaudiologia da Universidade Estadual Paulista - UNESP, Campus de Marília, no âmbito do Programa de Jovens Pesquisadores em Centros Emergentes de Pesquisa da Fundação de Amparo à Pesquisa do Estado de São Paulo - FAPESP; Doutora em Linguística pelo Instituto de Estudos da Linguagem - IEL - da Universidade Estadual de Campinas - UNICAMP.

(3) Fonoaudióloga; Doutora em Linguística pelo Instituto de Estudos da Linguagem - IEL - da Universidade Estadual de Campinas - UNICAMP.

Conflito de interesses: inexistente

\section{INTRODUÇÃO}

A Sequência de Pierre Robin (SPR), clinicamente bem definida ${ }^{1}$, é descrita na literatura como composta por uma tríade de anomalias caracterizadas por micrognatia, glossoptose e fissura do palato (FP), sendo que esta última se encontra presente na maioria dos casos e, em geral, é ampla e em forma de "U",3. Conforme descrito na literatura, a FP isolada ou quando associada às malformações adicionais, como é o caso da SPR, é considerada como fator de risco para alterações de fala severas ${ }^{4}$. Em geral, na presença de uma fissura de palato, recomenda-se que a cirurgia de palato 
(palatoplastia primária) seja realizada previamente ou ao redor do primeiro ano de vida, a fim de favorecer a produção adequada da fala. $\mathrm{Na}$ presença da SPR, nem sempre é possível o fechamento do palato por volta dos 12 meses devido à presença de obstrução respiratória, devendo a mesma ser indicada após estabilização do quadro respiratório ${ }^{5}$.

A presença de um problema estrutural como a FP (associada ou não à SPR), durante o primeiro e o segundo ano de vida da criança, pode favorecer o desenvolvimento de produções atípicas na região laríngea ou faríngea devido às dificuldades para gerar e/ou manter pressão aérea necessária para produção dos sons orais ${ }^{6,7}$. Estas produções podem ser entendidas como padrões gestuais aprendidos pela criança em resposta a estruturas do trato que estão comprometidos devido à FP e/ou DVF. Uma vez aprendida, estas produções, também designadas como articulações compensatórias (doravante $\mathrm{AC}$ ), passam a fazer parte do sistema fonológico das crianças, já que as mesmas utilizam essas AC na tentativa de marcar os contrastes fônicos da língua. Ressalta-se, porém, que mesmo na presença das $\mathrm{AC}$ há comprometimento severo na fala das crianças com FP, tornando difícil o entendimento até por familiares mais próximos.

Tal prejuízo na inteligibilidade de fala é resultado, principalmente, da perda efetiva de contraste especialmente de ponto de articulação, tendo em vista o sistema de oposições que a língua estabelece entre as consoantes. Na maioria das vezes, o ponto articulatório se encontra alterado (geralmente alterado para mais posterior) durante a produção da $A C$, sendo o modo de articulação mantido ${ }^{8,9}$. Dentre os vários tipos de $A C$ descritos na literatura ${ }^{9,10}$ o mais comumente observado na produção de crianças com FP é a oclusiva glotal, também denominada de "golpe de glote"10.

A oclusiva glotal é uma articulação transiente de natureza consonantal, que tem como correlato acústico um som, essencialmente, brusco ${ }^{8}$. Este tipo de AC é mais comum em substituição aos sons oclusivos (vozeados ou não) e é, em geral, considerado distinto da omissão de um segmento ${ }^{8,9}$. A oclusiva glotal é descrita como resultante da adução das pregas vocais, devido a um aumento de pressão aérea gerada na região subglótica e consequente tensão na região mais baixa do trato vocal, seguida de intensa abertura e fechamento das pregas vocais ${ }^{10}$. Nos casos em que há aumento significante de pressão e tensão para oclusão das pregas vocais, pode haver um deslocamento ou o encontro das pregas ventriculares, medialmente, obstruindo severamente a região laríngea, achados estes, observados, a partir de estudos ${ }^{10-13}$ que utilizaram a nasofaringoscopia. Uma variação à oclusiva glotal, a partir de dados da videofluorocopia, foi descrita ${ }^{10}$ como um movimento de elevação da laringe e posteriorização do dorso da língua em direção à faringe, com participação da epiglote para a oclusão.

A oclusiva glotal pode ser produzida em conjunto com a mesma oclusiva que a substitui, caracterizando uma articulação dupla ou uma co-produção'. A identificação clínica da presença desta AC é feita, fundamentalmente, com base no julgamento auditivo ${ }^{14,15}$ (e visual, no caso da co-produção). Apesar disso, as avaliações instrumentais, tais como nasoendoscopia e videofluoroscopia têm contribuído para uma melhor caracterização dessas produções, uma vez que essas avaliações permitem a observação direta das regiões laríngeas e/ou faríngeas. As informações advindas da análise acústica também têm auxiliado no entendimento da oclusiva glotal, uma vez que permitem inferir sobre os ajustes articulatórios realizados em tais produções. Alguns estudos descreveram, em termos de presença ou ausência dos parâmetros acústicos, informações sobre a oclusiva glotal. Nestas descrições, foram observadas as seguintes características acústicas: (1) ausência ${ }^{16}$ e presença da soltura da oclusiva glotal marcada acusticamente por uma ou mais espícula "spikes/transients"16-18, (2) espectro difuso, apresentando forma semelhante à das vogais vizinhas ${ }^{18}$ e (3) concentração de energia acústica de baixa energia, principalmente nas regiões de F1 e $\mathrm{F} 2^{17}$.

Embora seja indiscutível a contribuição das descrições acústicas para a identificação e/ou caracterização das oclusivas glotais, enquanto $A C$, essas informações são escassas na literatura, principalmente quando se tratam de crianças com oclusiva glotal, falantes do Português Brasileiro (PB) e, ainda, associada à SPR. Além disso, com relação à oclusiva glotal, não tem sido observado na literatura uma preocupação em identificar a presença de contrastes encobertos, e de discutir suas modificações acústicas e/ou articulatórias que podem não ser resgatadas auditivamente. Contudo, entende-se que os contrastes encobertos são indícios da criança para tentar marcar um determinado contraste fonológico da língua, fato que tem sido objeto de estudo em crianças falantes do PB com desenvolvimento típico e desviante da linguagem ${ }^{19}$, mas que não apresentam FP. A literatura internacional tem apresentado essa preocupação ao estudar a aquisição de linguagem de crianças sem $\mathrm{FP}^{20}$ e, também, com $\mathrm{FP}$, mas que apresentam a plosiva dorso médio palatal enquanto $A C^{21}$, identificadas pela eletropalatografia. Já na literatura nacional, destaca-se um estudo ${ }^{22}$ que evidenciou a presença de contrastes encobertos 
ao investigar o contraste da nasalidade de vogais e consoantes em falantes com fissura labiopalatina operada que apresentavam ressonância de fala hipernasal.

A hipótese adotada no presente estudo é a de que há variabilidade na produção da oclusiva glotal em 'substituição' às oclusivas velares (no caso / $/$ / e /g/) e que tal variabilidade sugere um contraste fônico encoberto para as consoantes velares surdas e sonoras, a fim de marcar um contraste na língua, a partir de produções distintas.

Desse modo, o presente estudo tem como objetivo caracterizar, a partir da análise acústica (espectral), produções julgadas auditivamente como oclusivas glotais em 'substituição' às oclusivas velares presentes na fala de uma criança com SPR e FP reparada cirurgicamente. Ainda, pretende-se verificar a existência ou não de contrastes encobertos nessas produções.

\section{APRESENTAÇÃO DO CASO}

Este trabalho foi realizado no Centro de Estudos da Educação e Saúde da UNESP, Campus de Marília, submetido e aprovado pelo Comitê de Ética em Pesquisa da Faculdade de Filosofia e Ciências (FFC), UNESP, Campus de Marília, sob o número 2011-35 e considerado sem risco. Participou deste estudo, uma criança de 5 anos, do sexo feminino, falante do Português Brasileiro (PB), com SPR e com FP corrigida cirurgicamente (palatoplastia primária) com 1 ano e 6 meses de idade. A participante deste estudo apresentava, na ocasião da coleta de dados, oclusiva glotal em substituição às oclusivas velares, de acordo com sua avaliação fonoaudiológica. Nenhum procedimento terapêutico com enfoque nessas consoantes foi realizado previamente à realização desse estudo. A criança não apresentava outros problemas na fala e ou auditivos na ocasião da coleta de dados desse estudo.

Os seguintes procedimentos foram realizados para caracterizar as oclusivas glotais apresentadas pela criança: (a) julgamento perceptivo-auditivo das produções por três fonoaudiólogas experientes na avaliação dos problemas de fala apresentados por sujeitos com fissura de palato e (b) análise acústica espectral destas produções.

\section{Coleta de dados}

O corpus utilizado para a coleta de dados perceptivos e acústicos foi composto por seis palavras dissílabas paroxítonas do PB ("capa, quibe, cuca" e "gato, guizo, gula") que combinavam as oclusivas velares em posição inicial de palavras com as vogais /i/, /a/, /u/ na posição acentuada. $O$ procedimento experimental consistiu em solicitar da criança cinco repetições de cada uma das palavras no interior da seguinte frase veículo: "Fale ___ bem bonito", por meio de uma atividade lúdica, computando um total de 30 ocorrências: 5 (repetições) $\times 3$ (vogais) $X 2$ (oclusivas) $=30$ ocorrências. $O$ uso da frase veículo teve como objetivo propiciar um maior controle da curva entoacional da produção de cada palavra do corpus, a fim de evitar a curva ascendente característica da produção obtida por meio de repetição isolada em forma de lista de palavras.

Um único avaliador conduziu as gravações da fala da criança, que foi gravada numa sala tratada acusticamente (Laboratório de Análise Acústica-LAAC, FFC, UNESP, Marília), utilizando equipamento digital de alta fidelidade (Gravador digital MARANTZ, microfone unidirecional Shure). As gravações foram feitas em $44 \mathrm{KHz}$ e todo material gravado foi analisado por meio do software PRAAT ${ }^{23}$. Após a obtenção das gravações foi realizado o julgamento perceptivo-auditivo das amostras de fala, com o objetivo de identificar a presença de oclusiva glotal nas consoantes velares (/k/ e/g/). Em seguida foi realizada a análise acústica dessas produções.

\section{Julgamento perceptivo-auditivo}

As frases constituídas das palavras de interesse foram julgadas por três fonoaudiólogos com pelo menos cinco anos de experiência na avaliação de problemas de fala associadas à fissura de palato. Os fonoaudiólogos foram instruídos a identificar apenas a presença ou não de oclusiva glotal, independentemente se outras compensações fossem percebidas.

O julgamento das frases foi realizado de forma aleatória e independente e pôde ser definido depois que os fonoaudiólogos ouvissem a gravação quantas vezes achassem necessário. A fim de verificar a consistência do julgamento (confiabilidade intra-juízes), seis amostras escolhidas aleatoriamente foram duplicadas, sendo uma com vogal /a/, uma com vogal /i/ e uma com a vogal /u/. Portanto, foram apresentadas para os fonoaudiólogos 36 frases ( 30 gravadas +6 duplicadas $=36$ frases para julgamento). As relações intra-juiz (6 frases) e inter-juízes (30 frases) da avaliação perceptivo-auditiva foram descritas, posteriormente, em porcentagem.

\section{Análise das características acústicas}

Primeiramente, foi realizada uma inspeção acústica da forma de onda aliada ao espectrograma, atentando-se para a presença de eventos não esperados durante a produção. Em seguida, os seguintes parâmetros fonéticos-acústicos, que marcam a produção de uma consoante oclusiva 
(closura, burst, transição formântica), foram analisados nesse estudo:

(1) parâmetros relativos às características espectrais da soltura (burst): pico espectral; momentos espectrais (centróide, variância, assimetria e curtose), intensidade relativa;

(2) parâmetros relativos às características acústicas das vogais adjacentes às oclusivas: medidas das frequências da transição formântica oclusivas/vogais (F1, F2 e F3) e trajetórias formânticas das vogais (F1, F2 e F3);

(3) parâmetros relativos ao padrão temporal: medidas de duração (absoluta e relativa) da closura, burst, transição formântica e voice onset time (VOT).

Informações detalhadas das descrições destes parâmetros foram reportadas previamente ${ }^{19}$.

Os resultados foram apresentados por meio de estatística descritiva (média, desvio padrão e intervalo de confiança - IC, que avaliou o quanto a média pode variar numa determinada probabilidade de confiança). Para verificar se os parâmetros de interesse diferiram entre as consoantes $/ \mathrm{k} / \mathrm{e} / \mathrm{g} /$ foi realizado o teste não paramétrico Wilcoxon. Para verificar se houve diferenças entre as vogais nas consoantes $/ \mathrm{k} / \mathrm{e} / \mathrm{g} /$ foi utilizado o teste não paramétrico Friedman, sendo que nos casos em que essas diferenças foram verificadas foi utilizado o teste não paramétrico Wilcoxon, para comparar as três vogais aos pares. Foram considerados estatisticamente significantes os resultados com valores de p inferiores a 0,05 $(p<0,05)$ e marginais os resultados com valores de $p$ entre 0,05 e 0,1 $(0,05<p$ $<0,1)$.

\section{RESULTADOS}

A concordância quanto à presença da oclusiva glotal foi de $100 \%$ para ambas as relações (intra e inter-juízes), o que indica que os juízes perceberam todas as produções (/k/ e $/ \mathrm{g} /$ ) como oclusivas glotais. Em uma inspeção mais geral dos dados, foi observada variabilidade dos parâmetros espectrais. Para o burst, as variações incluíram a ausência da marca típica da produção de um som oclusivo (fig. 1A), a presença de uma ou mais espículas (fig. 1B) e, ainda, a presença de uma discreta marca de ruído característico da soltura (burst) (Figura 1C). Quanto aos formantes de transição, foi observado desde a presença com maior ou menor duração (Figura 1C e 1B, respectivamente) até a ausência deste parâmetro (Figura 1A).

Ainda sobre a inspeção dos dados, porém com relação à ocorrência de variações entre as consoantes / $/ \mathrm{k} / \mathrm{e} / \mathrm{g} /$ nos parâmetros espectrais inspecionados; pôde-se avaliar que: de 15 produções do /k/ houve presença de burst em $87 \%$ e presença de formantes de transição em 67\%; e de 15 produções do $/ \mathrm{g} / 67 \%$ apresentaram burst e $80 \%$ formantes de transição. Essas variações também puderam ser computadas considerando as vogais separadamente.

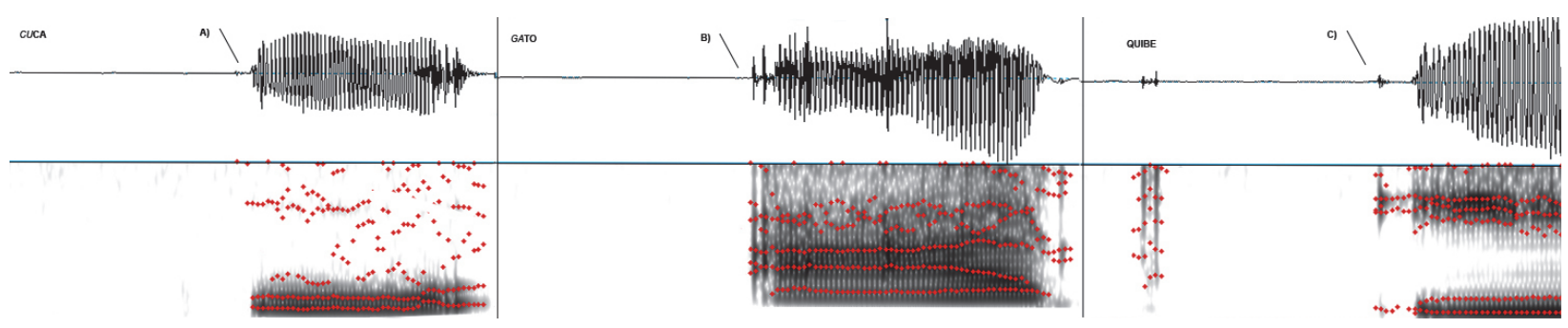

Figura 1 - Variações na forma de onda e no espectrograma durante produções percebidas como oclusiva glotal para /k/ e /g/. A) na primeira sílaba da palavra "cuca", observa-se que não há espículas nem formantes de transição. B) na primeira sílaba da palavra "gato", vê-se duas espículas próximas e discreta transição formântica. C) na primeira sílaba da palavra "quibe" há uma espícula distante do início da vogal e movimento mais marcado de formantes de transição.

De forma geral, observou-se que há uma tendência da produção das consoantes seguidas pela vogal /u/ ser mais afetada pela ausência dos parâmetros (burst e transição formântica) e da produção das consoantes seguidas pela vogal /a/ ser mais afetada pela presença destes mesmos parâmetros (burst e transição formântica) (Tabela 1). 
Tabela 1 - Ocorrência de burst e de transição formântica (TF) na produção das três vogais (a, i, u), para as consoantes $\mathrm{k} \mathrm{e} \mathrm{g}$

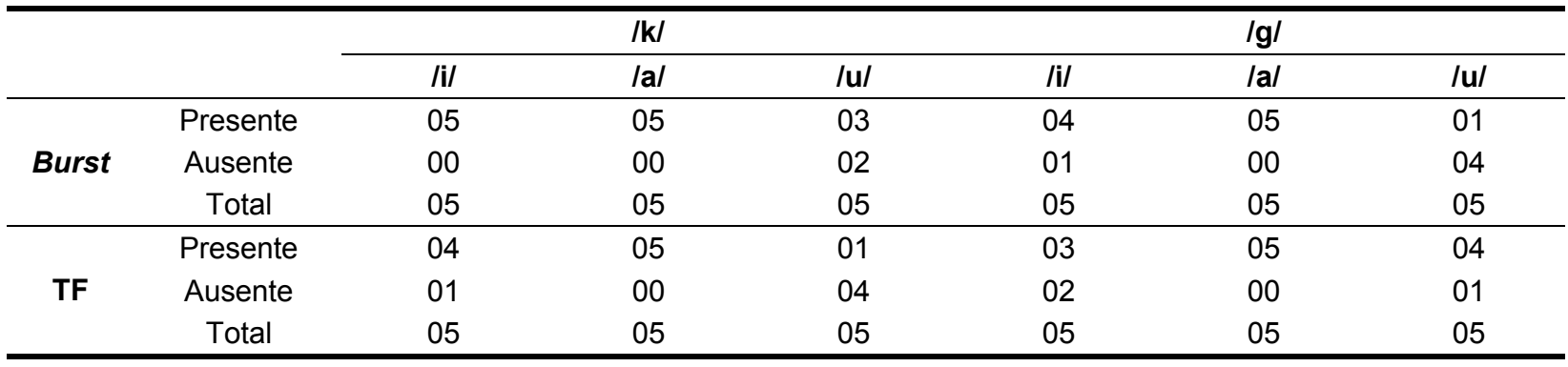

Em relação à análise acústica, ao se comparar os resultados obtidos para a diferença entre as duas consoantes velares $(/ \mathrm{k} / \mathrm{e} / \mathrm{g} /)$ foram observadas diferenças estatisticamente significantes nos parâmetros (a) espectral (burst), (b) temporal (VOT e duração relativa da oclusiva na palavra) e (c) relativos às características acústicas das vogais adjacentes às oclusivas (período estacionário de F3 $-p=0,015)$.
Com relação ao burst (Tabela 2) foram observadas diferença marginal no pico espectral $(p=0,096)$ e diferença estatisticamente significante em um de seus momentos espectrais: variância $(p=0,001)$. Ainda, houve diferença estatisticamente significante nas durações relativa e absoluta deste parâmetro burst.

Tabela 2 - Comparação das consoantes com a duração absoluta, em milissegundos (ms), duração relativa, em porcentagem (\%), frequência do pico, em Hertz $(\mathrm{Hz})$ e variância $(\mathrm{Hz})$ do burst

\begin{tabular}{|c|c|c|c|c|c|c|c|c|}
\hline & \multicolumn{2}{|c|}{ duração } & \multicolumn{2}{|c|}{$\%$ burst } & \multicolumn{2}{|c|}{ freq. pico } & \multicolumn{2}{|c|}{ variância } \\
\hline & $/ \mathbf{k} /$ & $\lg /$ & $/ \mathbf{k} /$ & $\lg /$ & $/ \mathbf{k} /$ & $\mid g /$ & $/ \mathbf{k} /$ & $\lg /$ \\
\hline Média (DP) & $15(10)$ & $9(7)$ & $7(5)$ & $4(4)$ & $\begin{array}{c}1954 \\
(1581)\end{array}$ & $\begin{array}{c}2046 \\
(1766)\end{array}$ & $\begin{array}{c}1536 \\
(1611)\end{array}$ & 797 (661) \\
\hline IC & 5 & 4 & 2 & 2 & 800 & 894 & 815 & 334 \\
\hline p-valor & \multicolumn{2}{|c|}{${ }^{*} 0,024$} & \multicolumn{2}{|c|}{${ }^{*} 0,022$} & \multicolumn{2}{|c|}{${ }^{\#} 0,096$} & \multicolumn{2}{|c|}{${ }^{*} 0,001$} \\
\hline
\end{tabular}

* valor significante $e^{*}$ valor marginal segundo teste de Wilcoxon para $\alpha=0,05$.

Ao se considerar a análise por vogais (Tabela 3 e 3.1), verificou-se que para (a) frequência do pico, as três vogais diferiram entre si em $/ \mathrm{k} /$, sendo essa diferença marginal em $/ \mathrm{g} /$, (b) variância, houve significância entre as vogais /i/ e /u/ e marginal para as demais, em $/ \mathrm{k} / \mathrm{e}$ as três vogais diferiram entre si, em /g/ e (c) centróide, as três vogais diferiram entre si para $/ \mathrm{k} / \mathrm{e}$, para $/ \mathrm{g} /$, a vogal $/ \mathrm{u} /$ foi a diferente. Nessa análise, encontrou-se ainda significância para intensidade e intensidade relativa (em ambos os casos para /k/ na vogal/a/). 
Tabela 3 - Comparação das três vogais (/i/, /a/, /u/) para /k/ e /g/ em frequência do pico, centróide e variância, em Hertz $(\mathrm{Hz})$, e em intensidade, em decibel (dB)

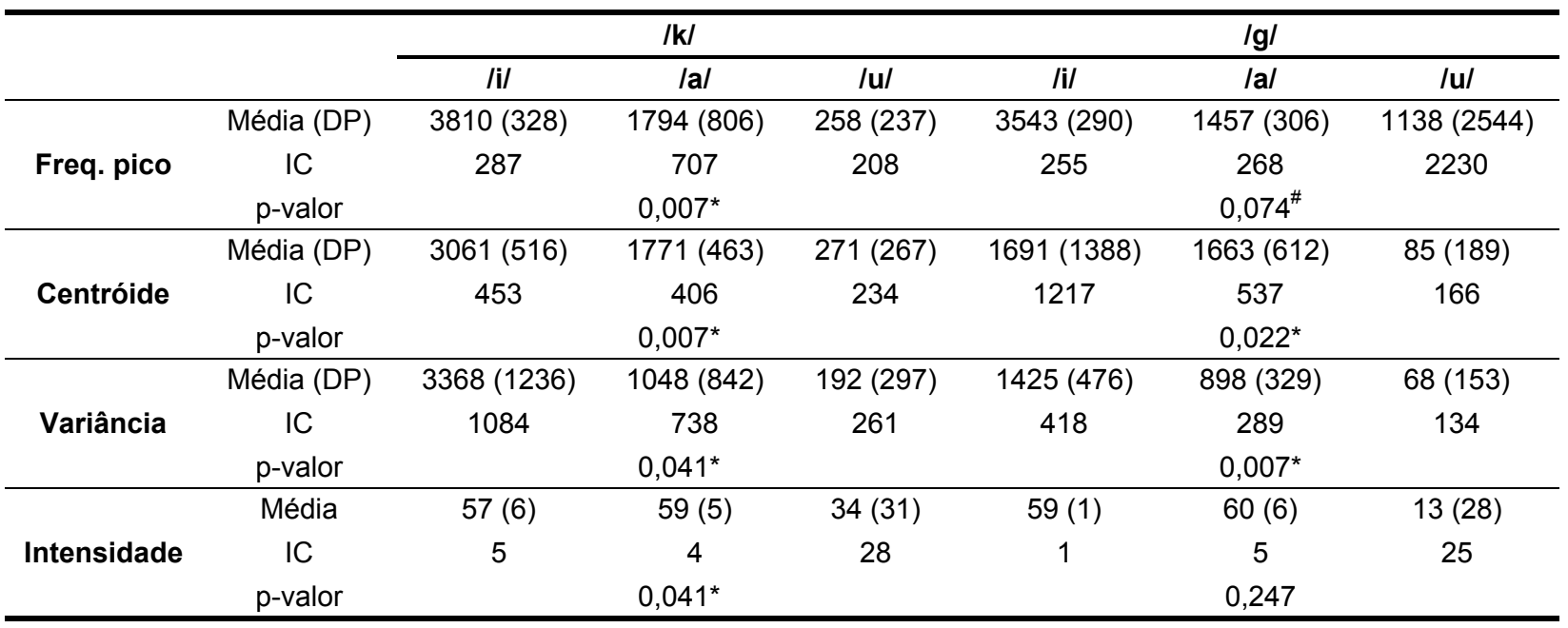

* valor significante $e^{\#}$ valor marginal segundo teste de Friedman para $\alpha=0,05$.

Tabela 3.1 - Comparação dos valores estatisticamente significantes da tabela 4 das três vogais (/i/, /a/, /u/) entre si, para / $/$ / e /g/, em frequência do pico, centróide e variância, em Hertz (Hz), e em intensidade, em decibel (dB)

\begin{tabular}{|c|c|c|c|c|}
\hline & & & lil & $|\mathbf{a}|$ \\
\hline \multirow{2}{*}{ Freq. pico } & \multirow{2}{*}{$/ \mathrm{k} /$} & $\mid \mathrm{a} /$ & $0,043^{*}$ & \\
\hline & & $\mid u /$ & $0,043^{*}$ & $0,043^{*}$ \\
\hline \multirow{4}{*}{ Centróide } & \multirow{2}{*}{$/ \mathrm{k} /$} & lal & $0,043^{*}$ & \\
\hline & & $\mid \mathbf{u} /$ & $0,043^{*}$ & $0,043^{*}$ \\
\hline & \multirow{2}{*}{$\lg /$} & lal & $0,893^{\#}$ & \\
\hline & & $/ \mathrm{u} /$ & $0,043^{*}$ & $0,043^{*}$ \\
\hline \multirow{4}{*}{ Variância } & \multirow{2}{*}{$/ \mathrm{k} /$} & $/ \mathrm{a} /$ & $0,080^{\#}$ & \\
\hline & & $\mid u /$ & $0,043^{*}$ & $0,080^{\#}$ \\
\hline & \multirow{2}{*}{$/ g /$} & $/ \mathbf{a} /$ & $0,043^{*}$ & \\
\hline & & lul & $0,043^{*}$ & $0,043^{*}$ \\
\hline \multirow{2}{*}{ Intensidade } & \multirow{2}{*}{$/ \mathrm{k} /$} & $\mid \mathbf{a} /$ & 0,225 & \\
\hline & & $/ \mathbf{u} /$ & $0,080^{\#}$ & $0,043^{*}$ \\
\hline
\end{tabular}

* valor significante $e^{\text {* }}$ valor marginal segundo teste de Wilcoxon para $\alpha=0,05$.

Já com relação ao VOT, houve diferença estatisticamente significante entre $/ \mathrm{k} / \mathrm{e} / \mathrm{g} /(\mathrm{p}=0,02)$, sendo que essa diferença estava no $/ \mathrm{g} /$ e na vogal $/ \mathrm{u} /$ (Tabela 4), quando as vogais foram consideradas na análise estatística.
Quanto ao parâmetro temporal "duração relativa da oclusiva na palavra", ao considerar as vogais para $/ \mathrm{k} /$ e $/ \mathrm{g} /$, separadamente, foram observadas diferenças estatisticamente significantes para o / $/ \mathrm{k}$ (na vogal /a/) e marginal para o/g/.

Tabela 4 - Comparação das três vogais (/i/, /a/, /u/) de /k/ e /g/, para VOT, em Hertz

\begin{tabular}{|c|c|c|c|c|c|c|c|c|c|}
\hline \multirow{2}{*}{ VOT } & \multicolumn{3}{|c|}{ /k/ } & \multicolumn{3}{|c|}{$\mid g /$} & \multirow{2}{*}{$\begin{array}{l}\text { p-valor } \\
\text { de } / g /\end{array}$} & \multicolumn{2}{|c|}{$\mid \mathbf{g} /$} \\
\hline & /i/ & $/ \mathrm{a} /$ & $/ \mathrm{u} /$ & /i/ & $\mid \mathrm{a} /$ & $/ \mathrm{u} /$ & & /i/ & $/ \mathrm{a} /$ \\
\hline Média (DP) & $24(11)$ & $20(7)$ & $12(11)$ & $15(6)$ & $17(7)$ & $2(4)$ & la/ & 0,686 & \\
\hline IC & 10 & $\begin{array}{c}6 \\
049\end{array}$ & 10 & 5 & 6 & 4 & $/ u /$ & $0,042^{\star *}$ & $0,039^{* *}$ \\
\hline
\end{tabular}

* valor significante segundo teste de Friedman para $\alpha=0,05 .{ }^{* *}$ valor significante segundo teste de Wilcoxon para $\alpha=0,05$. 
Em relação aos parâmetros relativos às vogais adjacentes, observaram-se diferenças estatisticamente significantes (a) nos dois primeiros formantes de transição (TF) - F1 e F2 (Tabela 5 e 5.1), (b) no período estacionário (PE) das vogais adjacentes (tabela 6 e 6.1) e (c) diferença relativa à transição (TF) e o período estacionário (PE) de F1 (para o / $/ \mathrm{k}$ / na vogal /a/).

Particularmente, para a TF F1 foi observada diferença estatisticamente significante em / $/$ / (para a vogal /a/) e em /g/ (para /a/ e marginal para as demais), e para a TF F2 a diferença observada foi significante para / $/ \mathrm{k}$ (entre /a/ e / $\mathrm{u} /$ e marginal para as demais) e foi marginal para /g/. Já para o PE de $\mathrm{F} 1$ houve diferença estatisticamente significante em /k/ na vogal /a/ (e marginal nas demais) e em /g/ na vogal /i/ (marginal nas demais); e, por fim, para PE de F2 e F3 a diferença foi significante para $/ \mathrm{k} / \mathrm{com}$ as três vogais diferindo entre si, e marginal para $/ \mathrm{g} /$.

Tabela 5 - Comparação das vogais (/l/, /a/, /u/) de /k/ e /g/, para transição formântica (TF) do primeiro formante (F1) e do segundo formante (F2), em Hertz (Hz)

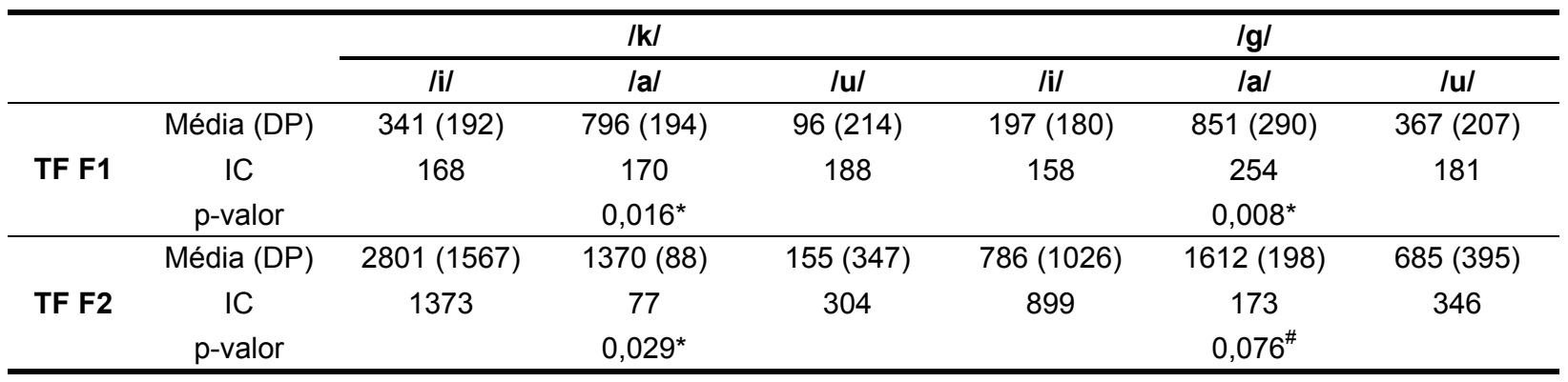

* valor significante $e^{\#}$ valor marginal segundo teste de Friedman para $\alpha=0,05$.

Tabela 5.1 - Comparação dos valores estatisticamente significantes da tabela 5 das três vogais (/i/, /a/, /u/) entre si, para /k/ e /g/, na transição formântica (TF) do primeiro formante (F1) e do segundo formante (F2), em Hertz (Hz)

\begin{tabular}{|c|c|c|c|c|}
\hline & & & lil & $|a|$ \\
\hline \multirow{4}{*}{ TF F1 } & \multirow{2}{*}{$/ \mathrm{k} /$} & $\mid a /$ & $0,043^{*}$ & \\
\hline & & $/ \mathrm{u} /$ & 0,144 & $0,043^{\star}$ \\
\hline & \multirow[b]{2}{*}{$/ g /$} & $\mid a /$ & $0,043^{*}$ & \\
\hline & & $/ \mathbf{u} /$ & $0,068^{\#}$ & $0,043^{\star}$ \\
\hline \multirow{2}{*}{ TF F2 } & \multirow{2}{*}{$/ \mathrm{k} /$} & $|a|$ & $0,080^{\#}$ & \\
\hline & & $/ \mathbf{u} /$ & $0,068^{\#}$ & $0,043^{*}$ \\
\hline
\end{tabular}

* valor significante $e^{*}$ valor marginal segundo teste de Wilcoxon para $\alpha=0,05$. 
Tabela 6 - Comparação das três vogais (/l/, /a/, /u/) adjacentes, para /k/ e /g/para /k/ e /g/ no período estacionário (PE) do primeiro formante (F1), segundo formante (F2) e do terceiro formante (F3), em Hertz (Hz)

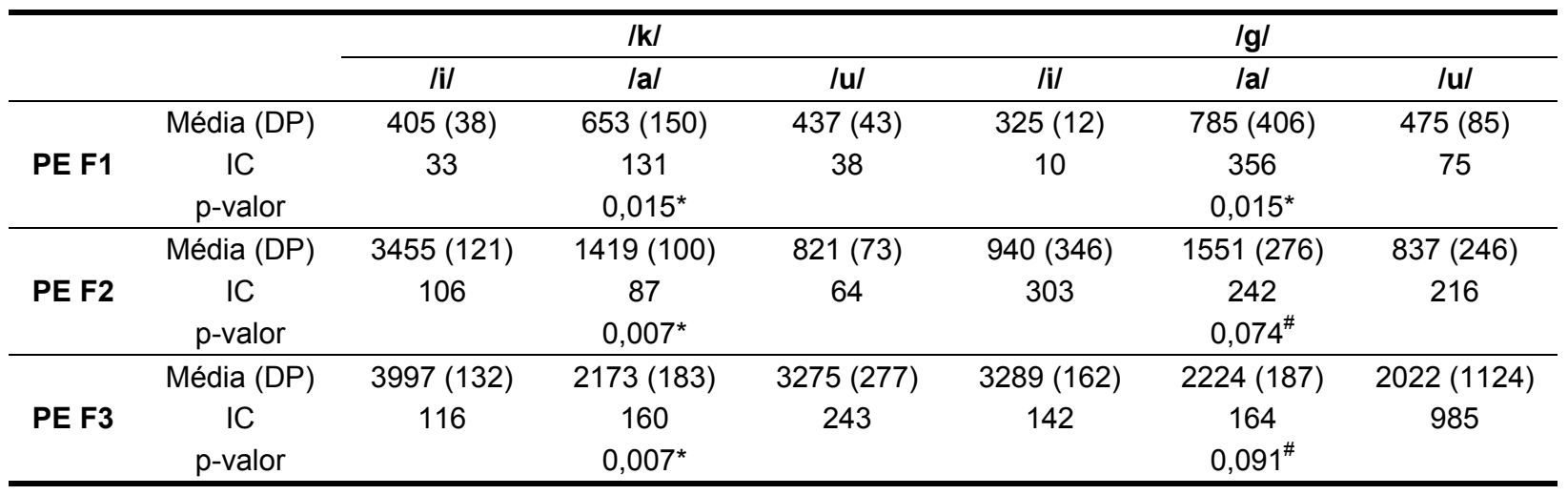

* valor significante $e^{\#}$ valor marginal segundo teste de Friedman para $\alpha=0,05$.

Tabela 6.1 - Comparação dos valores estatisticamente significantes da tabela 6 das três vogais (/i/, /a/, / $/$ /) adjacentes, para / $/ \mathrm{e} / \mathrm{e} / \mathrm{g} /$, no período estacionário (PE) do primeiro formante (F1), segundo formante (F2) e do terceiro formante (F3), em Hertz (Hz)

\begin{tabular}{|c|c|c|c|c|}
\hline & & & li/ & $|a|$ \\
\hline \multirow{4}{*}{ PE F1 } & \multirow{2}{*}{$/ \mathrm{k} /$} & $\mid a /$ & $0,043^{*}$ & \\
\hline & & $/ \mathrm{u} /$ & $0,078^{\#}$ & $0,043^{*}$ \\
\hline & \multirow{2}{*}{ lg/ } & $\mid \mathrm{a} /$ & $0,043^{*}$ & \\
\hline & & $\mid \mathrm{u} /$ & $0,043^{*}$ & $0,080^{\#}$ \\
\hline \multirow{2}{*}{ PE F2 } & \multirow{2}{*}{$/ \mathrm{k} /$} & $|\mathbf{a}|$ & $0,043^{*}$ & \\
\hline & & $\mid u /$ & $0,043^{*}$ & $0,043^{*}$ \\
\hline \multirow{2}{*}{ PE F2 } & \multirow{2}{*}{$/ \mathrm{k} /$} & $|\mathbf{a}|$ & $0,043^{*}$ & \\
\hline & & $\mid \mathbf{u} /$ & $0,043^{*}$ & $0,043^{*}$ \\
\hline
\end{tabular}

* valor significante $e^{\text {\# }}$ valor marginal segundo teste de Wilcoxon para $\alpha=0,05$.

\section{DISCUSSÃO}

A literatura, de uma forma geral, considera o julgamento perceptivo-auditivo como a base para uma avaliação da fala ${ }^{24}$ relacionada à FP associada ou não a outras malformações. A literatura defende que a decisão final sobre a presença dos problemas fala deve ser feita considerando o julgamento perceptivo-auditivo do avaliador, ainda que vários aspectos possam dificultar tal o julgamento, tais como a falta de concordância intra e inter-juízes e experiência do avaliador em identificar e utilizar procedimentos padronizados para transcrever foneticamente as $\mathrm{AC}^{24}$. No presente estudo, houve $100 \%$ de concordância intra e inter-juízes para as produções julgadas como oclusiva glotal. Estes resultados apontam para alta concordância intra e inter avaliadores, sugerindo que a experiência, as condições de gravação e as condições de apresentação das amostras para serem julgadas favoreceram a obtenção destes resultados. Estes aspectos, dentre outros, foram citados na literatura como importantes para serem levados em conta quando se trata de uma avaliação perceptivo-auditiva ${ }^{25}$.

Destaca-se que, embora todas as produções apresentadas para os juízes tenham sido percebidas como oclusivas glotais, as informações acústicas dos parâmetros analisados mostraram variabilidade. $\mathrm{Na}$ inspeção geral dos dados deste estudo, observou-se que as variações nas produções incluíram desde a presença de espículas até quase uma não aparente marca de ruído característico do burst. Ainda, os dados nem sempre revelaram os sinais acústicos que são esperados quando há uma produção glotal, conforme as prévias análises das características acústicas realizadas a partir dos arquivos de som do IPA - Alfabeto Fonético Internacional - (disponíveis em http://web.uvic.ca/ling/ resources/ipa/handbook_downloads.htm), a saber: silêncio absoluto na fase da closura seguido de 
soltura brusca (burst), marcado por uma espícula. Pelo fato de não termos no Português Brasileiro uma descrição acústica e/ou articulatória de oclusivas glotais (uma vez que esses sons não fazem parte do inventário fonético e fonológico dessa língua), a possibilidade de analisar estes arquivos de som do IPA, pelo software PRAAT, foi de fundamental importância neste estudo para que se tivesse uma referência dos parâmetros acústicos da produção glotal. No presente estudo, observou-se, em várias produções de $/ \mathrm{k} / \mathrm{e} / \mathrm{g} /$, a presença de uma ou mais espículas bem como ruído na closura, de forma semelhante às características acústicas das produções caracterizadas como oclusivas epiglotais, também conforme apresentados pelo IPA.

Além disso, nas produções epiglotais disponibilizadas nos arquivos de sons do IPA, foi observado movimento mais marcado de formantes de transição quando comparado com o mesmo movimento na oclusiva glotal, que se apresentou discreto ou inexistente. Essas variações também puderam ser observadas nos dados do presente estudo ao se considerarem as vogais separadamente, sendo que de forma geral, observou-se que a produção das consoantes seguidas pela vogal / $u /$ foi a mais afetada pela ausência dos parâmetros e que as consoantes seguidas pela vogal /a/foram as mais afetadas pela presença dos mesmos. Assim, observando os achados de inspeção geral de nosso estudo, pode-se se sugerir que a vogal /u/ favoreça uma produção glotal, enquanto que a vogal /a/ uma produção epiglotal, ao se levar em conta informações do IPA.

As singularidades nas produções observadas neste estudo demonstram variabilidade, assim como as descrições apresentadas na literatura sobre $\mathrm{FP}^{17-18}$ que resumem as produções glotais. Nos casos clínicos reportados, a descrição da soltura da oclusiva glotal é marcada acusticamente por uma ou mais espículas e espectro difuso, apresentando frequentemente forma semelhante à das vogais vizinhas. Considerando que os dados reportados na literatura ${ }^{17-18}$ trazem somente informações mais gerais sobre aspectos acústicos associados à AC do tipo oclusiva glotal (glottal stop), não revelando as particularidades da coleta de dados, a comparação dos achados entre os estudos torna-se mais difícil. No entanto, achados de inspeções da forma de onda muito semelhantes aos do presente estudo foram encontrados em adultos com $\mathrm{FP}^{16}$. Além disso, não se pode deixar de considerar que as informações acústicas aqui obtidas foram de uma criança com SPR, podendo então diferir daquela associada à FP sem alterações faciais adicionais.

Com relação aos parâmetros acústicos analisados para $/ \mathrm{k} /$ e $/ \mathrm{g} /$, sem levar em consideração as vogais, observou-se que para o burst, tanto a frequência (pico e variância) quanto a duração (absoluta e relativa) diferenciaram estas duas consoantes, com uma duração menor para /g/ que para $/ k /$, mostrando variabilidade entre estas consoantes. Quanto ao VOT, sua duração significantemente menor para $/ \mathrm{g} /$ que para $/ \mathrm{k} /$ sugere uma tendência do sujeito (mesmo antes da terapia) em diferenciar $/ \mathrm{k} /$ e /g/ da maneira esperada, já que na produção normal (sem compensação), geralmente, os sons vozeados possuem valores negativos de VOT $^{26,27}$ com essa mesma tendência observada para crianças em fase de aquisição fonológica ${ }^{28}$. Isso parece afetar a vogal adjacente à produção compensatória (oclusiva glotal) na altura do terceiro formante, já que as medidas extraídas no período estacionário da vogal (PE F3) apresentaram diferenças estatisticamente significantes entre $/ \mathrm{k} / \mathrm{e} / \mathrm{g} /$, sendo a menor média deste último. Assim, esses resultados apontam para uma tentativa da criança, em suas produções glotais, de contrastar estes dois segmentos $(/ \mathrm{k} / \mathrm{e} / \mathrm{g} /)$, ainda que tais diferenças não sejam resgatadas pelo ouvinte. Um clássico estudo $^{29}$ sobre o vozeamento contrastivo das oclusivas durante a aquisição de linguagem mostrou que as diferenças de VOT que são imperceptíveis aos ouvintes adultos são produzidos por algumas crianças. Ainda, outros estudos sobre aquisição fonológica normal ${ }^{20}$ e desviante, porém sem a presença de $\mathrm{FP}^{19}$ evidenciaram, com o auxílio de análise acústica, a presença de contrastes encobertos na fala das crianças investigadas, ressaltando a contribuição da análise acústica no entendimento de como se dá o estabelecimento de contrastes fônicos na língua. Na literatura direcionada à área da fissura labiopalatina, informações sobre a presença de contrastes encobertos na fala de sujeitos com FP também são reportadas, ainda que somente para a produção compensatória do tipo plosivo dorso médio palatal, identificadas com o apoio da eletropalatografia ${ }^{21}$

Particularmente, os parâmetros relativos às características espectrais da soltura (burst) que diferenciaram o evento laríngeo nos três contextos vocálicos investigados foram o pico espectral, centróide e a variância. Já quanto aos parâmetros relativos às características acústicas das vogais adjacentes à oclusiva, a transição formântica de F1 e F2 foram os parâmetros que diferenciaram as três vogais. Tais parâmetros (pico espectral, centróide, e transição formântica de F1 e F2), quando combinados, indicam variabilidade nas produções das frases incluindo as seis palavras de interesse ("quibe", "capa", "cuca", "guizo", "gato", "gula"). Ainda que estes parâmetros tenham comportamento diferente nas três vogais investigadas, as 
vogais /a/ e /u/ foram, na maioria das vezes, as que mais marcaram diferenças nos parâmetros investigados nas produções de / $/ \mathrm{k} / \mathrm{e} / \mathrm{g} /$, indicando que estas vogais são mais vulneráveis à presença da produção compensatória. Ainda, as diferenças observadas nas vogais foram mais marcadas na consoante $/ \mathrm{k} /$ do que $/ \mathrm{g} /$, sugerindo que a consoante velar não vozeada, conforme produzida pela criança deste estudo, é mais estável do que a consoante velar vozeada, ainda que ambos sejam ouvidas como compensatórias. Estes achados indicam, mais uma vez, a presença de contrastes encobertos para as consoantes / $/ \mathrm{k} /$ e /g/. Por fim, os resultados referentes ao período (estacionário $\mathrm{PE}$ ) das vogais adjacentes indicam que a produção compensatória glotal em substituição às consoantes velares afeta significantemente as vogais de seu entorno, extrapolando, portanto, os limites das consoantes, compreendido entre a oclusão e à transição formântica.

De forma geral, a análise dos dados obtidos permite inferir que a variabilidade observada nas produções da criança pode ser uma estratégia por ela utilizada para marcar contrastes na língua, ainda que os mesmos não tenham magnitude suficiente para serem resgatados auditivamente pelo ouvinte. Futuros estudos que considerem informações obtidas por meio da inspeção visual aliada ao cálculo de medidas acústicas em crianças com FP e, em especial, com história de SPR poderão favorecer ainda mais a possibilidade de investigar, naquelas produções consideradas como estáveis (porém, compensatórias), a presença de variabilidade que ainda não é suficientemente distintivos, por meio de percepção auditiva.

\section{CONCLUSÃO}

Os resultados obtidos no presente estudo indicaram concordância total dos juízes quanto à presença da oclusiva glotal (golpe de glote) em todas as produções da criança envolvendo as consoantes /k/ e /g/. Contudo, tanto a inspeção visual quanto a análise dos parâmetros acústicos estudados indicaram variabilidade nas produções. Tal variabilidade sugere que a criança realizou produções distintas para as consoantes $/ \mathrm{k} / \mathrm{e} / \mathrm{g} /$. Ainda sugere que estas consoantes também foram realizadas de forma distinta quando associadas às vogais adjacentes. De forma geral, a variabilidade observada nas produções da criança sugere tentativas da criança em marcar os contrastes da língua, ainda que com magnitude insuficiente para que os contrastes sejam resgatados pelos ouvintes. Estes achados revelam a importância em se atrelar a análise acústica à avaliação perceptivo-auditiva em estudos de crianças com FLP e, em especial, daquelas com SPR. Quando combinadas, as informações perceptivo-auditivas e acústicas podem propiciar um maior entendimento das produções realizadas pela criança, além de favorecer o direcionamento da fonoterapia.

\section{ABSTRACT}

The cleft palate, in association with Pierre Robin Sequence, may foster the development of atypical (compensatory) productions on the child's speech such as the use of glottal stop as a substitution for occlusive sounds (voiced or voiceless). Acoustic parameters of glottal stop for $/ \mathrm{k} /$ and $/ \mathrm{g} / \mathrm{produced}$ by a 5 year-old girl with cleft palate repaired in association with Pierre Robin Sequence were analyzed. For this study, we used six words consisting of velar stops in the initial word position combining the vowels /a/, /i/ e /u/ in the stressed position were selected. There was total agreement (100\%) as for the presence of the glottal stop for both intra and inter-judges. Inspection of the data via spectrogram showed variability of spectral parameters (burst and formant transition), and variations could also be computed, separately considering the vowels. Statistical analysis revealed a statistical difference between the two velar consonants $(/ \mathrm{k} /$ and $/ \mathrm{g} /$ ) in spectral (burst), temporal (VOT and duration of occlusion on the word) parameters and those relating to the acoustic features of vowels adjacent to stops (stationary period of F3. The acoustic features of glottal stop suggest that the child may have used strategies to set out phonic contrasts in the language, but these strategies did not have enough magnitude to be perceived by the listener.

KEYWORDS: Speech Acoustics; Spectrography; Speech Disorders; Cleft Palate; Pierre Robin Syndrome 


\section{REFERÊNCIAS}

1. Jakobsen LP, Knudsen MA, Lespinasse J, García Ayuso C, Ramos C, Fryns JP, et al. The genetic basis of the Pierre Robin Sequence. Cleft Palate Craniofac J. 2006; 43(2):155-9.

2. Evans AK, Rahbar R, Rogers GF, Mulliken JB, Volk MS. Robin sequence: a retrospective review of 115 patients. Int J Pediatr Otorhinolaryngol. 2006; 70 (6):973-80.

3. Butow KW, Hoogendijk CF, Zwahlen RA. Pierre Robin sequence: appearances and 25 years of experience with an innovative treatment protocol. $\mathrm{J}$ Pediatr Surg. 2009;44(11):2112-8.

4. Persson C, Elander A, Lohmander-Agerskov A, Söderpalm E. Speech Outcomes in Isolated Cleft Palate: Impact of Cleft Extent and Additional Malformations. The Cleft Palate-Craniofac J. 2002; 39(4): 397-408.

5. Souza TV, Marques IL, Carneiro AF, Bettiol $\mathrm{H}$, Freitas JAS. Nasophayngoscopy in Robin sequence: clinical and predictive value. Cleft Palate Craniofac J. 2003; 40(6):618-23.

6. Yamashita RP, Calais LL, Miguel HC, Trindade IEK. Avaliação da resistência laríngea em indivíduos portadores de insuficiência velofaríngea com distúrbio articulatório compensatório. Acta ORL. 2006; 21(2):263-7.

7. Oliveira RP. Nasalidade de crianças com sequencia de Robin após palatoplastia primária com as técnicas de Furlow ou Von Langenbeck. Tese (Doutorado-Área de Concentração: Distúrbios da Comunicação Humana) - Hospital de Reabilitação de Anomalias Craniofaciais. Universidade de São Paulo. 2009.

8. Kummer AW. Velopharyngeal dysfunction and resonance disorders. In: Kummer AW (ed). Cleft palate \& craniofacial anomalies: effects on speech and resonance. San Diego: Singular; 2001. p.145-76.

9. Trost-Cardamone JE. Diagnosis of specific cleft palate speech error patterns for planning therapy or physical management needs. In: Bzoch KR (ed). Communicative disorders related to cleft lip and palate. 4th ed. Austin: Pro-Ed; 2004. p. 313-30.

10. Witzel MA. Communicative impairment associated with clefting. In: Sphrintzen RJ, Bardach $\mathrm{J}(\mathrm{ed})$. Cleft palate speech management. Mosby: St. Louis; 1995.

11. Kawano M, Honjo I, Kojima $H$, Kurata $F$, Tanokuchi F, Kido N. Laryngeal constrictions on glottal stop in cleft palate speech. Studia Phonologica. 1991; XXV:7-13.

12. Kido N, Kawano M, Tanochushi F, Fujiwara $Y$, Honjo I, Kojima H. Glottal stop in cleft palate speech. Studia Phonologica 1992; XXVI:34-41.
13. Kido N, Kawano M, Tanokuchi F, Fujiwara $Y$, Kurata K, Kojima H, Honjo I. Glottal stop in cleft palate speech (2nd report): dynamic alterations of laryngeal movement during production of voiceless stop CV syllables. Studia Phonologica 1993; XXVII:33-41.

14. John A, Sell D, Sweeney T, Harding-Bell A, Williams, A. The cleft palate audit for speechaugmented: a validated and reliable measure for auditing cleft palate. Cleft Palate Craniofac J. 2006; 43:272-88.

15. Lohmander A, Persson,C. A longitudinal study of speech production in Swedish children with unilateral cleft lip and palate and two-stage palatal repair. Cleft Palate Craniofac J. 2008; 45(1), 32-41.

16. Lima-Gregio AM. Oclusiva glotal e laringalização em sujeitos com fissura palatina: um estudo segundo abordagem dinamicista [Tese]. Campinas (SP): Universidade Estadual de Campinas; 2011.

17. Philips BJ, Kent RD. Acoustic-phonetic descriptions of speech production in speakers with cleft palate and other velopharyngeal disorders. In: Lass NJ (ed). Speech and language: advances in basic research and practice. Orlando: Academic Press; 1984; 11:113-68.

18. Kent RE, Liss JM, Phillips BJ. Acoustic analysis of velopharyngeal dysfunction in speech. In: Bzoch KR (ed). Communication disorders related to cleft lip and palate. 4th ed. Austin: Pro-Ed; 1989.

19. Berti LC, Marino VCC. Contraste fônico encoberto entre /t/ e / $/ \mathrm{k}$ : um estudo de caso de normalidade e de transtorno fonológico. Revista CEFAC (no prelo) 2011.

20. Scobbie JM, Gibbon F, Hardcastle WJ, Fletcher $P$. Covert contrast as a stage in the acquisition of phonetics and phonology. In: Broe M, Pierrehumbert $\mathrm{J}$ editors. Papers in Laboratory Phonology V: Language Acquisition and the Lexicon. Cambridge: Cambridge University Press. 2000; p. 194-207.

21. Gibbon FE, Crampin L. An Elecropalatographic investigation of middorsum palatal stops in an adult with repaired cleft palate. Cleft Palate-Craniofac J. 2001; 38(2), 96-105.

22. Di Ninno CQMS. O contraste de nasalidade em falantes normais e com fissura palatina: aspectos da produção [tese]. Belo Horizonte: Universidade Federal de Minas Gerais - Faculdade de Letras; 2008.

23. Boersma $P$, Weenink D. Praat: doing phonetics by computer (Version 5.0.30) Avaliable from: http:// www.praat.org/ (Nov 2008).

24. Lohmander A, Olsson, M. Methodology for perceptual assessment of speech in patients with cleft palate: A critical review of the literature. Cleft Palate-Craniofacial Journal. 2004, 41(1): 64-70. 
25. Sell D, John A, Harding-Bell A, Sweeney T, Hegarty F, Freeman J. Cleft audit protocol for speech (CAPS-A): a comprehensive training package for speech analysis. Int J Lang Comm Dis. 2008;1-20. 26. Ögüt $F$, Kiliç M, Engin E, Midilli R. Voice onset time for turkish stop consonants. Speech Commun. 2006; 48:1094-9.

27. Barroco MAL, Domingues MTP, Pires MFMO, Lousada M, Jesus LMT. Análise temporal das oclusivas orais do Português Europeu: um estudo de caso de normalidade e perturbação fonológica. Rev CEFAC. 2007; 9(2):154-63.

28. Bonatto MTRL. A produção de plosivas por crianças de três anos falantes do Português Brasileiro. Rev CEFAC. 2007; 9(2):199-206.

29. Macken MA, Barton D. The acquisition of the voicing contrast in English: a study of voice onset time in word-initial stop consonants. Journal of Child Language. 1980; 7:41-74.

Endereço para correspondência:

Viviane Castro Marino

Rua Santa Helena, 1967 casa 07

Jardim Estoril - Marília - São Paulo

CEP: 17410-514

E-mail: vivianemarino2 @yahoo.com.br 